\title{
Non-separable Solutions of Helmholtz' equation revisited
}

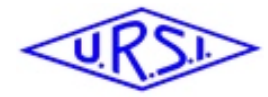

\author{
Emmanuel H. Van Lil ${ }^{(1)}$, Pieter J. Luypaert ${ }^{(1)}$ \\ (1) KU Leuven, div. ESAT-Telemic, Kasteelpark Arenberg, 10 - box 2444, B-3001 Heverlee, \\ E-mail: Emmanuel.VanLil@ESAT.KULeuven.Be
}

\begin{abstract}
Even if the idea is already very old (proposed first by Moseley in 1965), it offers analytical solutions of Helmholtz equations for curved waveguides. One of the key elements in their successful use is the computation of recursion formulas to find a numerical solution to the problem of analysis of generally shaped waveguides. They will be derived in this paper. Even if the original theory includes a generalisation to 3 dimensional cavities we will limit ourselves to 2 dimensional waveguide cases.
\end{abstract}

\section{Introduction}

The non-separable solutions are found by starting from the classical solution, given by the well known solutions consisting of a product of sines and cosines, The easiest derived functions consist in subtracting 2 separable ones to obtain the correct solution for a triangular waveguide. By taking a derivative with respect to the wave number components, we obtain the more general form of those functions [1]. We will first derive the recursion formulas in section 2. Section 3 will be devoted to the synthesis of curved waveguides with analytically know solutions, while section 4 will be devoted to the analysis of waveguides of a given shape.

\section{Derivation of the recursion formulas}

The standard separable solutions are all of the shape:

$$
\psi_{0}=\sin \left(\xi x+\varphi_{x}\right) \sin \left(\eta y+\varphi_{y}\right)
$$

with $\xi^{2}+\eta^{2}=k^{2}-\zeta^{2}, k$ being the wave number in the medium (assumed linear, isotropic and homogeneous), and $\zeta$ the wave number in the longitudinal direction. Higher order functions are obtained by taking the total derivative with respect to $\xi$ and multiplying with $\eta$. So,

$$
\psi_{l}=H^{l} \psi_{0}=\left(\eta \frac{d}{d \xi}\right)^{l} \psi_{0}=\left(\eta \frac{\partial}{\partial \xi}-\xi \frac{\partial}{\partial \eta}\right)^{l} \psi_{0}
$$

This first expression is completely equivalent with the right more classical operator containing partial derivatives [2].

In the original papers, analytical expressions for those higher-order functions were given up to order 2, and orders up to 4 were worked out in a Ph. D. thesis and displayed for order 2 also in $[3,4]$. However, by expanding in a similar way as in [2] the general expression

$$
\begin{aligned}
& \psi_{l}=f_{1}^{l} \sin \left(\xi x+\varphi_{x}\right) \sin \left(\eta y+\varphi_{y}\right) \\
& +f_{2}^{l} \sin \left(\xi x+\varphi_{x}\right) \cos \left(\eta y+\varphi_{y}\right) \\
& +f_{3}^{l} \cos \left(\xi x+\varphi_{x}\right) \sin \left(\eta y+\varphi_{y}\right) \\
& +f_{4}^{l} \cos \left(\xi x+\varphi_{x}\right) \cos \left(\eta y+\varphi_{y}\right)
\end{aligned}
$$

leads us to a recursion formula to compute $f_{i}^{l+1}$ if all 4 $(\mathrm{i}=1,2,3,4) \quad f_{i}^{l}$ are known. Starting from $f_{1}^{0}=1$ and $f_{2}^{0}=f_{3}^{0}=f_{4}^{0}=0$ we can then easily compute the $(\mathrm{x}$, $\mathrm{y}, \xi$ and $\eta$ dependent) functions $f_{i}^{l}$ with the following recursion formula:

$$
f_{i}^{l+1}=H f_{i}^{l}=(-1)^{u} \eta x f_{w}^{l}+(-1)^{v} \xi y f_{s}^{l}
$$

The indices $u, v, w$ and $s$ are defined as follows:

$u=i+1-5$ floor $(i / 4)$

$v=5-i-5$ floor $[(5-\mathrm{i}) / 4]$

$w=2$ floor $[(2+i) / 2]-3$ floor $[(i+1) / 4]-$ floor $(i / 4)$

$s=5-2$ floor $[(2+i) / 2]+3$ floor $[(i+1) / 4]+$ floor $(i / 4)$

or similar formulas, since they have only to be defined for $i=1 . .4$. Splitting the 4 cases leads to the most efficient way of programming formula (4). We still have to compute the first term in the right-hand side of (4). Fortunately we can do this by applying the operator $H$ up to $l+1$ times::

$$
\begin{aligned}
& H^{k} f_{i}^{l+1}=H^{k+1} f_{i}^{l}+(-1)^{u} x \sum_{m=0}^{k} C_{k}^{m} H^{m}(\eta) H^{k-m} f_{w}^{l} \\
& +(-1)^{v} y \sum_{m=0}^{k} C_{k}^{m} H^{m}(\xi) H^{k-m} f_{s}^{l}
\end{aligned}
$$

with $C_{k}^{m}$ the binomial coefficients and

$$
\begin{aligned}
& H^{m}(\eta)=\eta \cos (\mathrm{m} \pi / 2)-\xi \sin (\mathrm{m} \pi / 2) \\
& H^{m}(\xi)=\xi \cos (\mathrm{m} \pi / 2)+\eta \sin (\mathrm{m} \pi / 2)
\end{aligned}
$$

Since all values of $H^{k} f_{i}^{0}$ are 0 , except for $f_{1}^{0}=1$, is it now possible to compute all values of $f_{1}^{l}$ and hence $\psi_{l}$ for any value of $\mathrm{x}, \mathrm{y}, \xi$ and $\eta$.

\section{Synthesis of waveguides}

This is the most easy part, even if this looks contradictory at first sight. One just has to chose the appropriate constant coefficients of an expansion of the kind 


$$
\psi(x, y)=\sum_{l=0}^{+\infty} O_{l} \psi_{l}(\xi, \eta, \mathrm{x}, y)
$$

To plot those fields easily, it is useful to be able to compute also all partial derivatives $\frac{\partial}{\partial x}, \frac{\partial}{\partial y}, \frac{\partial^{2}}{\partial x^{2}}, \frac{\partial^{2}}{\partial x \partial y}, \frac{\partial^{2}}{\partial y^{2}}$. Since the operator $H$ is commutative with all those partial derivatives (since it does not contain $\mathrm{x}$ or $\mathrm{y}$ ), one can use the same recursion formulas for those derivatives, of course starting with different initial values i.e. :

$$
\begin{aligned}
& H^{m} f_{3 x}^{0}=\xi \cos (\mathrm{m} \pi / 2)+\eta \sin (\mathrm{m} \pi / 2) \\
& H^{m} f_{2 y}^{0}=\eta \cos (\mathrm{m} \pi / 2)-\xi \sin (\mathrm{m} \pi / 2)
\end{aligned}
$$

all other values of $H^{m} f_{i x}^{0}$ or $H^{m} f_{i y}^{0}$ being zero. The extra subscript $\mathrm{x}$ or $\mathrm{y}$ being the coefficients of a similar expansion as (3), but for the derivative function with respect to $\mathrm{x}$ and $\mathrm{y}$.

For the analysis of waveguides, we will also need derivatives with respect to the wave numbers $\xi$ and $\eta$. Since those are more tricky, they will be discussed in section 4 .

A few examples of tangential $E$ field lines for a $\mathrm{TM}_{11}$ waveguide with only 2 expansion functions, 0 and 2, but with different coefficients $O_{2}\left(O_{0}=1\right)$ are given in Fig. 1 and Fig. 2, resp. for $O_{2}=0.005$ and $O_{2}=0.015$. Note that the second contour (green lines for 0.005 ) is also a valid boundary, with the first (black lines for 0.005) as inner contour.

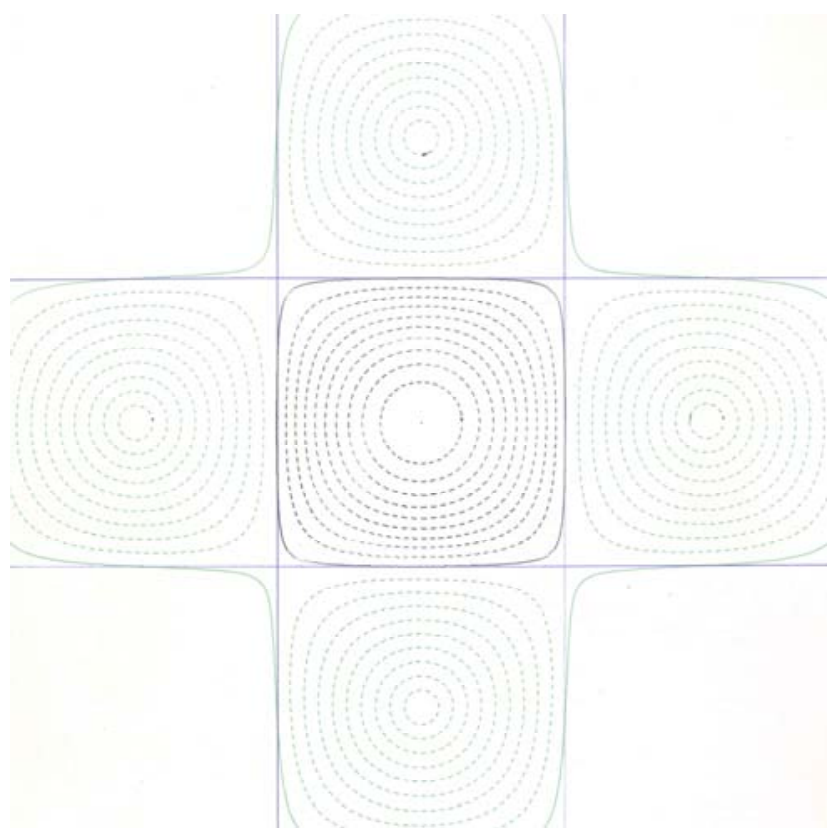

Fig. 1: Example of E field lines in cavities with $\mathrm{O}_{2}=0.005$, starting from the $\mathrm{TM}_{11}$ mode.

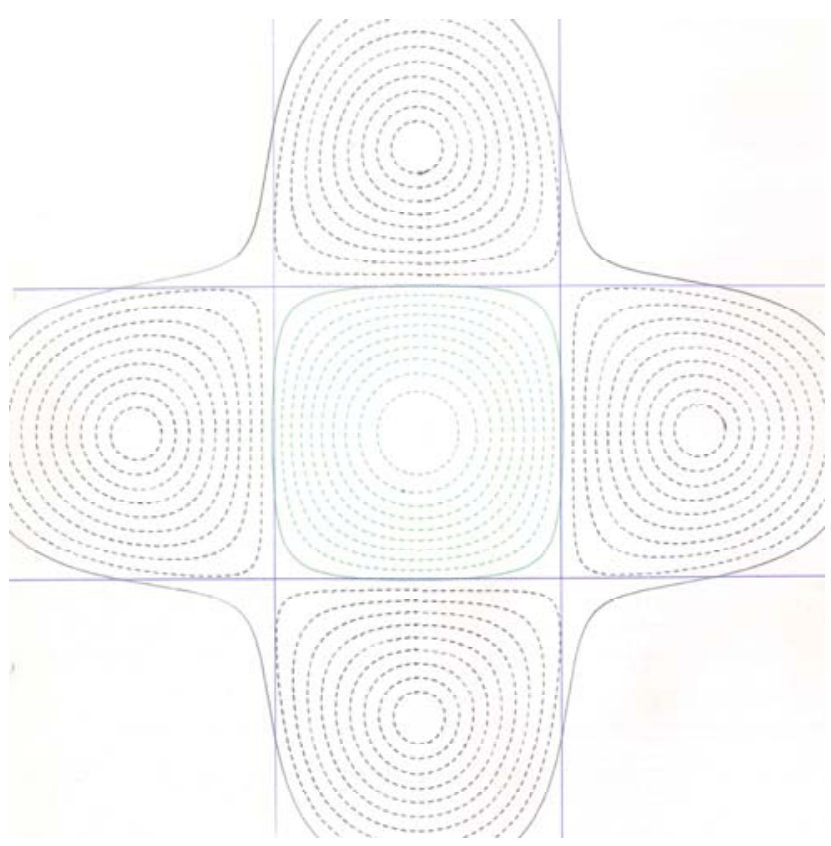

Fig. 2: Example of E field lines in cavities with $\mathrm{O}_{2}=0.015$, starting from the $\mathrm{TM}_{11}$ mode.

If we increase the size of the coefficient $\mathrm{O}_{2}$ to 0.095 , the second contour becomes much larger and tries to follow the bisectors like in Fig. 3.

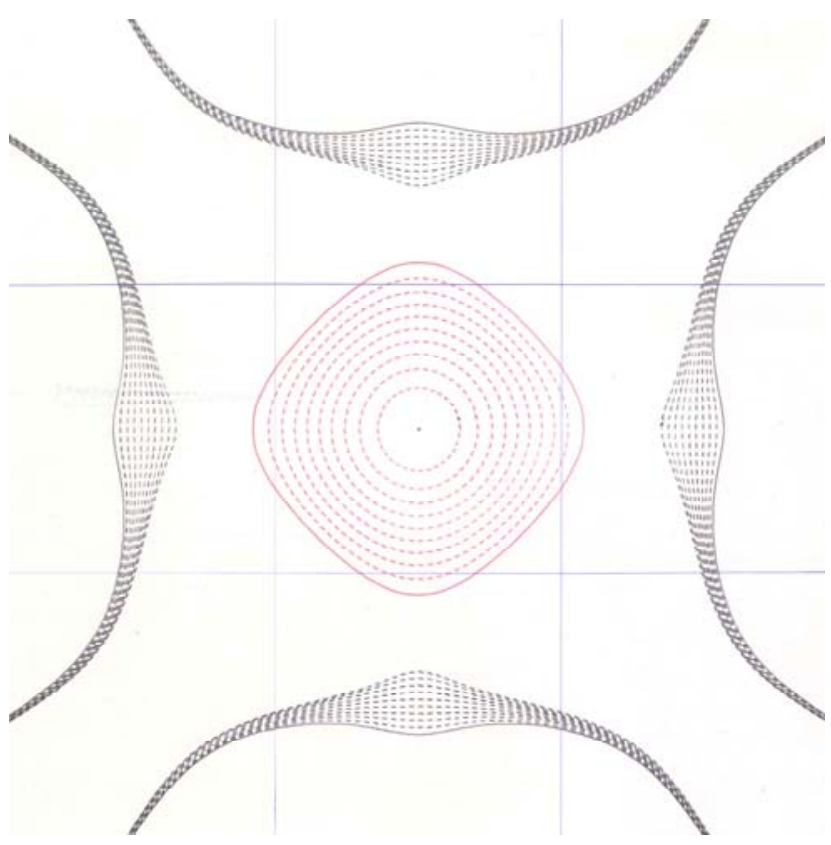

Fig. 3: Example of E field lines in cavities with $\mathrm{O}_{2}=0.095$, starting from the $\mathrm{TM}_{11}$ mode.

There is even a value above which no simple contour can be found. This maximal value depends on the proportion between the $\mathrm{x}$ and $\mathrm{y}$ dimensions (compression factor of the ellipse) and is shown in Fig. 4. 


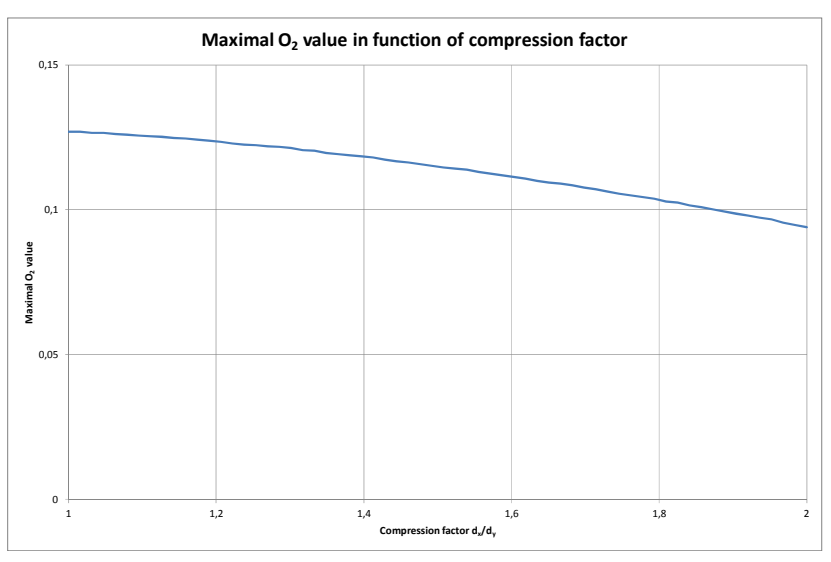

Fig. 4: Maximum values of $\mathrm{O}_{2}$ for $\mathrm{TM}_{11}$ modes.

\section{Analysis of waveguides}

This is in fact the most difficult part. Indeed, we have to minimize a cost function consisting of the expansion on the given boundary. We have chosen to use a least-square minimisation of the function:

$$
\int_{\alpha}^{\beta}\left|\mathrm{g}^{\prime}(\mathrm{q}, \xi, \eta)-\psi^{\prime}(\mathrm{q}, \xi, \eta)\right|^{2} d q=\text { Minimal }
$$

where $\alpha$ and $\beta$ are points on the given cross-section of the waveguide and $\mathrm{q}$ is a running coordinate on this contour (like the length). Normally the contour is closed, so $\alpha=\beta$, even if for numerical purposes and with enough symmetry, we may reduce the contour to a part of the boundary of the waveguide (like 1/4 for the cases shown). Let us consider first the $\mathrm{TM}_{\mathrm{mn}}$ modes. In this case we can consider the scalar functions in (7). as being the longitudinal electric field $E_{z}$ and use just the expansions investigated in the previous sections. This implies:

$$
\begin{aligned}
& g^{\prime}(x, y, \xi, \eta)=-\psi_{0}(x, y, \xi, \eta) \\
& \psi^{\prime}(x, y, \xi, \eta)=\sum_{1}^{l_{t}} O_{l} \psi_{l}(x, y, \xi, \eta)
\end{aligned}
$$

The problem to be solved consists in computing the $l_{t}$ coefficients $O_{l}$ as well as the values of the wave numbers $\xi$ and $\eta$, that best fit to the contour in question (remember that for the square waveguide $\xi=m \pi / d_{x}$ and $\eta=n \pi / d_{y}$ ). Necessary conditions to minimize the integral in (7) are:

$$
\begin{aligned}
& \frac{\partial}{\partial O_{l}} \int_{\alpha}^{\beta}\left|\mathrm{g}^{\prime}(\mathrm{q}, \xi, \eta)-\psi^{\prime}(\mathrm{q}, \xi, \eta)\right|^{2} d q=0 \\
& \frac{\partial}{\partial \xi} \int_{\alpha}^{\beta}\left|\mathrm{g}^{\prime}(\mathrm{q}, \xi, \eta)-\psi^{\prime}(\mathrm{q}, \xi, \eta)\right|^{2} d q=0 \\
& \frac{\partial}{\partial \eta} \int_{\alpha}^{\beta}\left|\mathrm{g}^{\prime}(\mathrm{q}, \xi, \eta)-\psi^{\prime}(\mathrm{q}, \xi, \eta)\right|^{2} d q=0
\end{aligned}
$$

This can be solved using a Newton-Raphson iteration formula. Also, this is why we need derivatives with respect to $\xi$ and $\eta$ up to second order.
We will only write out the first derivative with respect to $\xi$. For the other derivatives $\frac{\partial}{\partial \eta}, \frac{\partial^{2}}{\partial \xi^{2}}, \frac{\partial^{2}}{\partial \xi \partial \eta}, \frac{\partial^{2}}{\partial \eta^{2}}$, a similar procedure applies. When taking the derivative of (5) with respect to $\xi$, we obtain:

$$
\begin{aligned}
& \frac{\partial}{\partial \xi} H^{k} f_{i}^{l+1}=\frac{\partial}{\partial \xi} H^{k+1} f_{i}^{l} \\
& -(-1)^{u} x \sum_{m=0}^{k} C_{k}^{m} \sin (m \pi / 2) H^{k-m} f_{w}^{l} \\
& +(-1)^{u} x \sum_{m=0}^{k} C_{k}^{m} H^{m}(\eta) \frac{\partial}{\partial \xi} H^{k-m} f_{w}^{l} \\
& +(-1)^{v} y \sum_{m=0}^{k} C_{k}^{m} \cos (m \pi / 2) H^{k-m} f_{s}^{l} \\
& +(-1)^{v} y \sum_{m=0}^{k} C_{k}^{m} H^{m}(\xi) \frac{\partial}{\partial \xi} H^{k-m} f_{s}^{l}
\end{aligned}
$$

To solve the system, we have to invert a matrix of size $l_{t}$ +2 iteratively, more specially:

$$
\left(I_{i j}\right)\left(\begin{array}{l}
O_{l} \\
\Delta \xi \\
\Delta \eta
\end{array}\right)=\left(I_{i}\right)
$$

with $i$ and $l \leq l_{t}+2$. Some integrals are easy and similar to a moment method matrix (note that the $\psi_{l}^{\prime}$ are not orthogonal, so that the matrix is a full matrix), like:

$$
\begin{aligned}
& I_{i l}=\int_{\alpha}^{\beta} \psi_{i}^{\prime}(\mathrm{q}, \xi, \eta) \psi_{l}^{\prime}(\mathrm{q}, \xi, \eta) d q \\
& I_{i}=\int_{\alpha}^{\beta} g^{\prime}(\mathrm{q}, \xi, \eta) \psi_{l}^{\prime}(\mathrm{q}, \xi, \eta) d q
\end{aligned}
$$

for $i$ and $l \leq l_{t}$. The elements surrounding this square part of size $l_{t} \mathrm{x} l_{t}$ are much more complex to write down and require indeed the derivatives with respect to the wave numbers.

For starting values of the iteration, we use $\xi=2.34 / d_{x}$ and $\eta=2.34 / d_{y}$ if the sizes of the waveguides are given in $\mathrm{m}$ The first $O_{l}$ 's are solved from using the starting values for . $\xi$ and $\eta$. By iterating, we notice the evolution of $\xi$, $\eta$ and the coefficient $O_{2}$ for a $\mathrm{TM}_{11}$ mode analysed with $l_{t}=2$ in

Table $\mathbf{1}$ (all examples in this section pertain to a square original guide of $9 \mathrm{~m} \times 9 \mathrm{~m}$ and the wave numbers are in $1 / \mathrm{m})$. Since a Newton Raphson is used, the convergence is fast . After 3 iterations we notice a maximal deviation of $5.55 \times 10^{-4}$ and a RMS deviation of $2.161 \times 10^{-6}$. The maximal deviation and the RMS deviation are defined as follows:

$$
\begin{aligned}
& \max \left(\left|\mathrm{g}^{\prime}(\mathrm{q}, \xi, \eta)-\psi^{\prime}(\mathrm{q}, \xi, \eta)\right|\right) \alpha \leq q \leq \beta \\
& \int_{\alpha}^{\beta}\left|\mathrm{g}^{\prime}(\mathrm{q}, \xi, \eta)-\psi^{\prime}(\mathrm{q}, \xi, \eta)\right|^{2} d q
\end{aligned}
$$


Table 1: Convergence for the $\mathrm{TM}_{11}$ mode, $\mathrm{O}_{2}$

\begin{tabular}{|l|l|l|l|}
\hline Iterat. \# & $\xi=\eta$ & $\mathrm{O}_{2}$ & RMS Error \\
\hline 0 & .1906667 & .06249836 & 0.128 \\
\hline 1 & .1889159 & .06249849 & $1.824 \times 10^{-3}$ \\
\hline 2 & .1889408 & .06249848 & $2.545 \times 10^{-6}$ \\
\hline 3 & .1889408 & .06249848 & $2.161 \times 10^{-6}$ \\
\hline
\end{tabular}

With 4 (in fact 2 since, due to symmetry, $O_{1}=O_{3}=0$ ) coefficients the improvement is nearly only noticeable in the invisible digits (see Table 2). After 4 iterations we notice a maximal deviation of $3.43 \times 10^{-7}$ and a RMS deviation of $2.161 \times 10^{-6}$.

Table 2: Convergence for the $\mathrm{TM}_{11}$ mode, $\mathrm{O}_{2}, \mathrm{O}_{4}$

\begin{tabular}{|c|c|c|c|l|}
\hline $\begin{array}{c}\text { Iter. } \\
\#\end{array}$ & $\xi=\eta$ & $\mathrm{O}_{2}$ & $\mathrm{O}_{4}$ & RMS Error \\
\hline 0 & .1906667 & .07812499 & .9765618 & 0.128 \\
\hline 1 & .1889160 & .07812499 & .9765618 & $1.821 \times 10^{-3}$ \\
\hline 2 & .1889409 & .07812499 & .9765618 & $3.831 \times 10^{-7}$ \\
\hline 3 & .1889409 & .07812499 & .9765618 & $8.372 \times 10^{-13}$ \\
\hline 4 & .1889409 & .07812499 & 9765618 & $8.223 \times 10^{-13}$ \\
\hline
\end{tabular}

For $\mathrm{TE}_{\mathrm{mn}}$ modes, the procedure is more complex. Indeed, in that case the obtained function is the magnetic field in the longitudinal direction $H_{z}$ and the functions

$$
\begin{aligned}
& g^{\prime}(x, y, \xi, \eta)=-\chi_{E} \psi_{0}(x, y, \xi, \eta)-\frac{\partial}{\partial n} \psi_{0}(x, y, \xi, \eta) \\
& \psi^{\prime}(x, y, \xi, \eta)=\sum_{1}^{l_{t}} O_{l}\left[\chi_{E} \psi_{l}(x, y, \xi, \eta)+\frac{\partial}{\partial n} \psi_{l}(x, y, \xi, \eta)\right]
\end{aligned}
$$

the derivative having to be taken according to the normal to the contour of the cross-section of the waveguide surface and the $\chi_{E}$ being proportional with the surface impedance (normally equal to 0 for a Perfectly Electrically Conducting surface), and depends on the material properties of the inner parts of the waveguide, as well as the longitudinal wave number:

$$
\chi_{E}=\frac{j\left(\varsigma^{2}-\omega^{2} \mu \varepsilon\right)}{\omega \mu} Z_{s}
$$

Table 3: Convergence for the $\mathrm{TE}_{11}$ mode, $\mathrm{O}_{2}$

\begin{tabular}{|l|l|l|l|}
\hline Iterat. \# & $\xi=\eta$ & $\mathrm{O}_{2}$ & RMS Error \\
\hline 0 & .1906667 & .026701 & .26238 \\
\hline 1 & .1889159 & .027350 & .15804 \\
\hline 2 & .1889407 & .027349 & $.34260 \times 10^{-2}$ \\
\hline 3 & .1889407 & .02349 & $.22317 \times 10^{-3}$ \\
\hline
\end{tabular}

Table 4: Convergence for the $\mathrm{TE}_{11}$ mode, $\mathrm{O}_{2}, \mathrm{O}_{4}$

\begin{tabular}{|c|c|l|c|l|}
\hline Iterat. \# & $\xi=\eta$ & \multicolumn{1}{|c|}{$\mathrm{O}_{2}$} & $\mathrm{O}_{4}$ & RMS Error \\
\hline 0 & .1906667 & .042958 & -.0421008 & .2897 \\
\hline 1 & .1889160 & .2088434 & -.0591003 & .01952 \\
\hline 2 & .1889410 & .294560 & -.0591011 & $6.036 \times 10^{-4}$ \\
\hline 3 & .1889410 & .293360 & -.0591011 & $7.873 \times 10^{-5}$ \\
\hline
\end{tabular}

\section{Conclusions}

We have derived in this paper all recursion formulas required to easily plot and synthesise waveguides with uncommon curved shapes with a perfect accuracy for the synthesis case.

Also analysis of given shapes was performed with an excellent accuracy allowing for instance to compute circular or elliptical waveguides without the need for more classical functions like Mathieu or Bessel functions. However, the method is not fool-proof for all cases. Indeed, there exists a maximum value of the coefficients above which no closed contour in the case of the synthesis can be found, as discussed in section 3 .

\section{Acknowledgements}

Part of this research was founded by the Belgian National Science Foundation.

\section{References}

1. D. S. Moseley, Non-separable solutions of the Helmholtz wave equation, Quart. Appl. Math. 22, 1965, pp. 354-357.

2. P. J. Luypaert and D. H. Schoonaert, On the synthesis of Waveguides and Cavities Realized with Nonseparable Solutions of Helmholtz Wave Equation, IEEE trans. MTT, 23, \#12, 1975, pp. 10611064.

3. P. J. Luypaert, A. R. Van de Capelle A., E. H. Van Lil E. and W. Ranson, Approximation of the field components of TE and TM-modes in waveguides and cavities with general cross section. , European Microwave Conference, Paris , 1978, pp. 423-427

4. P.J. Luypaert, A.R. Van de Capelle, E. Van Lil. Approximation of Mathieu functions by nonseparable solutions of Helmholtz wave equation in elliptic waveguides and cavities. Colloquium on Microwave Communication, Budapest, 1-4 August 1978. 